\title{
Underwater dual-magnification imaging for automated lake plankton monitoring
}

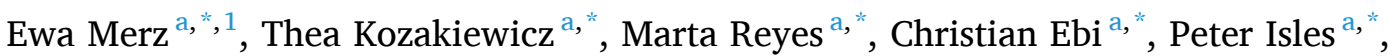 \\ Marco Baity-Jesi ${ }^{\text {a, }}$, Paul Roberts ${ }^{\text {b, }}$, Jules S. Jaffe ${ }^{\text {, }}$, Stuart R. Dennis ${ }^{\text {a }}$, Thomas Hardeman ${ }^{\text {a }}$, \\ Nelson Stevens ${ }^{a}$, Tom Lorimer ${ }^{\mathrm{a}, \mathrm{b}}$, Francesco Pomati ${ }^{\mathrm{a}, *, 1}$ \\ ${ }^{a}$ Swiss Federal Institute of Aquatic Science and Technology (Eawag), Ueberlandstrasse 133, 8600 Dübendorf, Switzerland \\ ${ }^{\mathrm{b}}$ Scripps Institution of Oceanography, University of California San Diego,9500 Gilman Drive, La Jolla, CA 92093-0238, United States \\ ${ }^{\mathrm{c}}$ Monterey Bay Aquarium Research Institute (MBARI), 7700 Sandholdt Road, Moss Landing, CA 95039, United States
}

\section{A R T I C L E I N F O}

\section{Keywords:}

Phytoplankton

Zooplankton

Size

Microscopy

Classification

Diversity

\begin{abstract}
A B S T R A C T
The Dual Scripps Plankton Camera (DSPC) is a new approach for automated in-situ monitoring of phyto- and zooplankton communities based on a dual magnification dark-field imaging microscope. Here, we present the DSPC and its associated image processing while evaluating its capabilities in i) detecting and characterizing plankton species of different size and taxonomic categories and ii) measuring their abundance in both laboratory and field applications. In the laboratory, body size and abundance estimates by the DSPC significantly and robustly scaled with measurements derived by microscopy. In the field, a DSPC installed permanently at $3 \mathrm{~m}$ depth in Lake Greifensee (Switzerland) delivered images of plankton individuals, colonies, and heterospecific aggregates at hourly timescales without disrupting natural arrangements of interacting organisms, their microenvironment or their behavior. The DSPC was able to track the dynamics of taxa, mostly at the genus level, in the size range between $\sim 10 \mu \mathrm{m}$ to $\sim 1 \mathrm{~cm}$, covering many components of the planktonic food web (including parasites and potentially toxic cyanobacteria). Comparing data from the field-deployed DSPC to traditional sampling and microscopy revealed a general overall agreement in estimates of plankton diversity and abundances. The most significant disagreements between traditional methods and the DSPC resided in the measurements of zooplankton community properties. Our data suggest that the DSPC is better equipped to study the dynamics and demography of heterogeneously distributed organisms such as zooplankton, because high temporal resolution and continuous sampling offer more information and less variability in taxa detection and quantification than traditional sampling. Time series collected by the DSPC depicted ecological succession patterns, algal bloom dynamics and diel fluctuations with a temporal frequency and morphological resolution that was never observed by traditional methods. Access to high frequency, reproducible and real-time data of a large spectrum of the planktonic ecosystem expands our understanding of both applied and fundamental plankton ecology. We conclude the DSPC is robust for both research and water quality monitoring and suitable for stable long-term deployments.
\end{abstract}

\section{Introduction}

\section{Abbreviations}

DSPC dual-magnification Scripps plankton camera

MIC inverted microscopy

0p5x $\quad 0.5$ times magnification

$5 \mathrm{p} 0 \mathrm{x} \quad 5.0$ times magnification

CNN convolutional neural network
Plankton are a key component of global ecology that play important roles in carbon and nutrient cycling as well as providing essential services to human society (e.g. clean water and fisheries) (Behrenfeld et al., 2001; Falkowski, 2012). Plankton, or "drifters", is a collective term for all organisms that inhabit aqueous ecosystems that are unable to control

\footnotetext{
* Corresponding authors.

E-mail addresses: ewa.merz@eawag.ch (E. Merz), francesco.pomati@eawag.ch (F. Pomati).

1 These authors contributed equally.
} 
their horizontal position in the water column. Hence, plankton comprises both prokaryotes and eukaryotes. Phytoplankton are the planktonic photosynthetic autotrophs and the primary producers of the pelagic (open water) ecosystem, while zooplankton comprises consumers that can be either herbivores, carnivores, or both. Most phytoplankton clades contain mixotrophic taxa, which combine photosynthesis with phagotrophic uptake of prey (usually bacteria or small protists). The phytoplankton are composed of an enormous variety of taxa, shapes and sizes (they span four orders of magnitude in size, from roughly $1 \mu \mathrm{m}$ to $1 \mathrm{~mm}$, overlapping with the zooplankton size range). While a single zooplankton sample in a lake may consist of several tens of taxa, the phytoplankton inventory of a lake may comprise hundreds of them.

Their short lifespans and strong sensitivity to environmental conditions make plankton effective indicators of environmental change and ecosystem health, and they are used to assess water and aquatic ecosystem quality worldwide (Xu, 2001; Directive 2000/60/EC). Information about plankton biomass and community composition, including variation among key functional traits, is essential to judge the state of ecological systems according to the Water Framework Directive in Europe (Directive 2000/60/EC). Plankton biodiversity surveys are also crucial to evaluate the resilience to change of ecosystem processes, extinctions and invasions, and to manage ecosystem services (Bartley et al., 2019; Cardinale et al., 2012; Pomati et al., 2011). Assessing plankton community composition is critically important for the monitoring and forecasting of harmful algal blooms (events of algal overgrowth that are dominated by toxic species). In freshwater ecosystems, cyanobacterial blooms cause large ecological and economical damage (Huisman et al., 2018), and have been increasing worldwide due to the effects of land use and climate change (Chorus and Bartram, 1999; Ho et al., 2019).

Given the importance of plankton for ecosystem processes and services, there is not only scientific interest but also a societal need and a policy requirement for monitoring plankton communities at low cost and ideally in real-time. Initiatives such as the Water Framework Directive in Europe demand that policymakers achieve "good status" for all water bodies and secure drinking water supplies for the population, requiring member states to consistently monitor aquatic biodiversity, including plankton communities and indicator species (Directive 2000/60/EC). The assessment of plankton community composition and taxa relative abundance is, however, the most difficult, timeconsuming and expensive aspect (Pomati et al., 2011). Dedicated laboratories use skilled and trained taxonomists to identify and count microalgae and zooplankton manually, sometimes taking several hours to count a sample. Taxonomists frequently misclassify items, count some objects more than once or overlook others (MacLeod et al., 2010) and mistakes are not traceable back in time. In many monitoring programs, microscope counts are only completed weeks or months after samples are collected, and are therefore unable to provide information in a timely, actionable manner. Sample collection, transport and storage also demand proper infrastructure and personnel time. Aquatic ecosystem monitoring would hence tremendously benefit from an automated, reliable, standalone system for plankton identification, classification and counting that produces transparent and reproducible results (not dependent on personal judgment), in near real-time, and that does not require sample collection and storage (additional costs).

Several automated quantitative tools for plankton counting have been established for use in the laboratory (e.g. Flow-cam, flow-cytometry, microscopy-imaging, Zooscan) and in the field (e.g. fluorescence probes, Cytobuoy, Flowcytobot, imaging cameras) (reviewed in Lombard et al., 2019). Laboratory-based tools can automate counting and sorting, but still require sample collection and processing, which changes the nature of the sample and requires time, resources and infrastructure. Field applications are more promising, but they too have limitations. Fluorescence sensors measuring chlorophyll-a, phycocyanin, or other photosynthetic pigments are common and widely deployed in the field, but they are unable to resolve taxonomic distinctions beyond coarse levels, and pigment content within and across species can vary widely, making estimates of abundances and biomass difficult (Johnson and Martiny, 2015). Automated in situ flow-cytometry has shown encouraging results for high-frequency monitoring of phytoplankton community dynamics and morpho-physiological traits (Fontana et al., 2018; Hunter-Cevera et al., 2016; Pomati et al., 2011; Sosik et al., 2010). Flow-cytometry complemented by water physics and chemistry data, and machine learning for analysis demonstrated the potential of high-frequency information for modeling environmental responses and predicting phytoplankton dynamics (Thomas et al., 2018). The dynamics of single species of phytoplankton and microzooplankton can be tracked and studied with flow-cytometry if assisted by imaging (Hunter-Cevera et al., 2016; Lombard et al., 2019). Still, flow-cytometers require constant maintenance and calibration and may disrupt natural spatial aggregates while sampling. Additionally, they have limited dynamic range in terms of size, often being able to follow the dynamics of a single trophic level only (Lombard et al., 2019). For phytoplankton particularly, high-frequency data of herbivore zooplankton could be essential to study variation in top-down controls as a response to environmental change (Murphy et al., 2020).

Here we describe a monitoring approach based on dark-field underwater imaging, by means of a dual-magnification camera derived from the Scripps Plankton Camera (SPC) system (Orenstein et al., 2020). It was designed to be a flexible, easily configurable imaging system that can observe objects from 10s of microns to several millimeters, and sample at high temporal frequency, with minimal influence on the fluid being imaged. Similar cameras have been successfully deployed in marine systems (Murphy et al., 2020; Orenstein et al., 2020), but never deployed before in freshwater environments. In this study, we compared the DSPC to traditional microscopy and high-frequency fluorescence-based monitoring using both field and laboratory comparisons. We then used this tool to observe plankton dynamics and trait distributions (e.g. size) across the whole planktonic food web in-situ, in the eutrophic lake Greifensee, with high temporal resolution (days, hours). Our results promote underwater imaging as an auspicious approach to generate empirical, high-resolution plankton time series.

\section{Methods}

\subsection{Description of the dual magnification camera}

\subsubsection{Instrument's design}

The Plankton Camera (DSPC) used in this study is a dualmagnification dark field underwater microscope, based on the Scripps Plankton Camera (SPC) system (Orenstein et al. 2020). When compared to the original SPC, the DSPC has several new features designed specifically to target plankton monitoring in freshwater. First, it is a dual-magnification microscope, extending the size range that can be photographed by overlapping spatial resolution (Fig. 1). Based on our trials, the highest magnification (5p0x - 5x magnification) has a detection range between $\sim 10 \mu \mathrm{m}-150 \mu \mathrm{m}$, while the lower magnification (0p5x - 0.5x magnification) ranges between $\sim 100 \mu \mathrm{m}-1 \mathrm{~cm}$. These estimates depend on the features of the imaged objects (e.g. color, transparency) and therefore are not fixed. The imaged volumes per frame for the two magnifications are (from high resolution to blob detection): $5 \mathrm{p} 0 \mathrm{x}=0.2-10 \mu \mathrm{L}, 0 \mathrm{p} 5 \mathrm{x}=4-200 \mu \mathrm{L}$, respectively. Second, internal batteries allow autonomous measurements for up to 3 hours without recharging, which is needed for manual depth profiling or sampling of remote sites (i.e. lakes, ponds). Finally, the distance between light sources and objective has been reduced to $5 \mathrm{~cm}$ to shorten the illumination path length and allow transmission of light in turbid waters (e.g. during thick algal blooms).

The DSPC is compact enough for one person to carry ( $57 \mathrm{~cm} \mathrm{~L} \mathrm{x} 19 \mathrm{~W}$, $\sim 27.2 \mathrm{~kg}$ weight) and is composed of two housings, one with the LED 

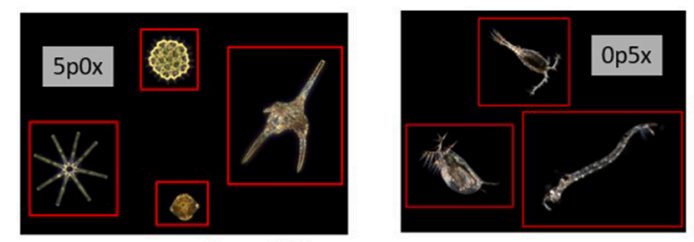

Focal Plane

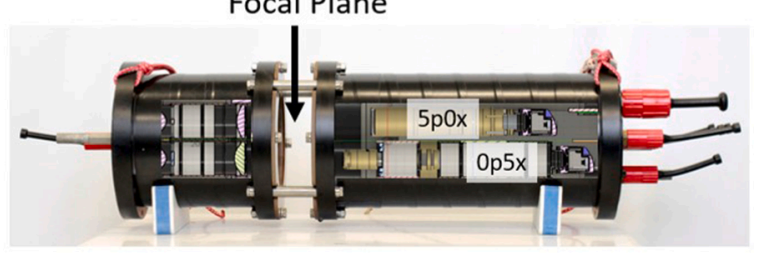

Light source
Fig. 1. Structural drawing of the dual-magnification plankton camera, showing LED light source points, the two cameras with different magnification. The focal plane is where water flows through and objects are imaged. The instrument captures full-frame pictures and automatically performs object detection (red boundaries around objects), cuts out these regions of interest (ROIs) that are stored, while full frames are usually discarded (Orenstein et al., 2020). Objects depicted as ROIs are only for display (not to scale).

light sources and another with the controlling hardware, cameras and lenses (Fig. 1). The instrument has no moving parts; therefore, it has low maintenance requirements (only cleaning of external surfaces). Housings have facing acrylic viewports that allow transmitting light from the LED to the microscopes. Water flows freely between the two viewports. To reduce fouling, our system was complemented with a UV lamp (https://amloceanographic.com/biofouling-control/) installed facing the acrylic viewports (Fig. 1). The onboard computer uses a Linux-based operating system. The camera works automatically, there is no need for regular calibration and, if installed in a monitoring platform like the DSPC at Lake Greifensee, pictures can be available in real-time, if a power supply, computer, and internet are available. The acquisition rate for pictures can be easily modified. The maximum rate is 10 frames per second (recording of videos is also possible). The actual specifics of the instrument can be found in Supplemental Table S1. The configurations of the machine used for imaging are reported in the Methods section relative to laboratory and field application.

\subsubsection{Image processing}

We use a modified version of the original Python SPCConvert image processing script (Orenstein et al., 2020), which converts raw regions of interest (ROIs) from the plankton camera into image mosaics and extracts statistics per processed sample and features of detected objects. The process consists of color conversion, edge-detection and segmentation, morphological feature extraction, foreground masking and inverse filtering of the masked foreground. The results are then saved into a new directory and a web app (HTML file) is built from the data showing converted pictures. The program creates a spreadsheet with 64 features (e.g. area, aspect ratio, max. length or orientation, color features, and more) of every detected object. The original Python code can be found here: https://github.com/tooploox/SPCConvert.

Because of their complex shape and moveable appendages, measuring body size in zooplankton is not straightforward: often zooplankton can be much wider than they are long when antennas are included (e.g. copepods), meaning that the major axis cannot reliably be used. Moreover, the presence of predators and other stressors also influences the overall shape of zooplankton and can induce the growth of defensive structures (e.g. spines, helmets, and elongated tails) further complicating the estimation of body size (Tollrian and Drew Harvell, 1999). To overcome these complexities, we developed The Daphnia Ruler (https://github.com/nelstevens/The-Daphnia-ruler), which effectively removes these appendages from calculations of zooplankton body size. Using the same edge detection concepts as SPCconvert, the outline of the zooplankton is refined by repeatedly eroding and dilating the binary image with round structuring elements of varying sizes, until a threshold solidity is reached indicating that all protuberances have been sufficiently removed. The resulting ellipse can then be treated as the core body and measured as for any other spheroid planktonic object.

\subsection{Annotation of images and classification based on deep learning}

Many labeled pictures are needed to create a training database for an automatic classification based on deep learning. We utilized a free and open-source platform, Taxonify, which expedites the process of species annotation. Through this platform, one can rapidly label pictures to taxon level and can add additional attributes to the pictures, e.g. colonies, dividing or parasites present. More information can be found on https://www.taxonify.org, and software codes are freely available at https://github.com/tooploox/taxonify_gui. For this study, we annotated a variable amount of images per class of interest (mainly zooplankton, in the $0 \mathrm{p} 5 \mathrm{x}$ magnification), ranging from a minimum of 10 (Chaoborus) to a maximum of 3321 (Dinobryon) (Supplemental Table S2). We then developed an automated deep learning classifier for objects detected by the 0p5x magnification of the DSPC. The model we used was a convolutional neural network (CNN), trained to distinguish 34 different classes of zooplankton, phytoplankton and other suspended solids (junk) (Supplemental Table S2). In the SI text, we describe the used CNN and its hyperparameters, and we show a series of validation benchmarks. We provide access to the code and a ready-to-use release of the classifier, at the following address: https://github.com/mbaityje/plankifier/releases/tag/v1.1.1. The default code settings contain all hyperparameter values used in this study.

\subsection{Laboratory calibration of DSPC imaging}

To use the DSPC in the laboratory, the space between the two viewports (Fig. 1) was covered with a black rubber sheet and flooded with water. Tissue culture flasks containing the imaged samples were manually held in the focal plane of the DSPC between the viewports to take measurements. For cultures where the sedimentation rate was high, we mixed the sample every 30 seconds with a Pasteur pipette through the lid of the flask.

\subsubsection{Calibration of plankton body size estimates}

We compared body size estimates for small ( $5 \mathrm{p} 0 \mathrm{x})$ and large $(0 \mathrm{p} 5 \mathrm{x})$ plankton organisms by the DSPC, featuring automated object detection and features extraction, to body size measurements done with images taken by inverted microscopy, using manual object detection and feature extraction using ImageJ (https://imagej.nih.gov/ij/). Having information on only two dimensions, size was evaluated as the area of objects rather than volume. For the comparison, only ROIs by DSPC that were comparable to manual microscopy (i.e. not cropped and imaged from the frontal plane) were selected and used for feature extraction (i.e. object area). Body size was estimated from DSPC images using SPC convert scripts (see Section 2.1.2). Taxon-specific mean body size was calculated by taking an average area in pixels overall saved objects, both for DSPC and microscopy.

5p0x magnification. We used the following phytoplankton cultures: Aphanizomenon flos aquae, Cyclotella meneghiniana, Scenedesmus acuminatus, Tetraedron minimum, Eudorina unicocca, Lagerheimia hindakii, Staurastrum punctulatum, Cosmarium botrytis, Oocystis solitaria, Planktothrix rubescens, Cystodinium sp, Volvox aureus, Peridinium sp. All cultures were grown in WC medium (Guillard, 1975) under standard conditions $\left(20^{\circ} \mathrm{C}\right.$ and 10:14 light:dark cycle) for at least 2 weeks. Cultures of two small zooplankton were also imaged at 5p0x magnification, the protist Euglena sp. and the rotifer Cephalodella sp. These were grown under standard conditions and with a protozoan pellet medium (provided by CarolinaTM, Biological Supply Company, Burlington NC, USA). A 
volume of 2-10 $\mathrm{mL}$ (depending on initial culture densities) was inoculated into a $25 \mathrm{~cm}^{2}$ tissue culture flask (vented cap, sterile, VWR 743-2311) and topped with tap water to a final volume of $60 \mathrm{~mL}$. 0p5x magnification. We used the two large protists Blepharisma sp. Spirostomum sp., which were grown under the same conditions as the protists used for 5p0x calibration. We also used living samples of Copepods sp., Daphnia sp., Keratella quadrata, Keratella cochlearis and Kellicotia sp. taken from Lake Greifensee (Switzerland).

Traditional microscopy. An aliquot of the imaged sample was taken and photographed with a light inverted microscope (Leica DMi8 magnification $\mathrm{x} 160$ or $\mathrm{x} 320$, depending on the size of the plankton species). Using ImageJ, the maximum and minimum axis lengths (perpendicular) were manually measured for 50 to 200 individuals and then used to calculate the object area by applying an ellipses formula.

\subsubsection{Calibration of DSPC object detection relative to plankton density}

We compared the average number of objects detected per frame (i.e. per second) in the DSPC to density measurements obtained by inverted microscopy for small (5p0x) and stereomicroscopy for large (0p5x) plankton organisms, in serial dilutions. This DSPC metric is not sensitive to frame rate and we tested its comparability to volumetric measurements.

5p0x magnification. The following cultures were used: Scenedesmus acuminatus, Oocystis solitaria, Tetraedron minimum, Botryococcus braunii, Euglena sp. and Cephalodella sp. For this experiment, we used culturing flasks (as in the previous experiment). An initial sample of 0.5 to $10 \mathrm{~mL}$ of the culture was taken and topped to $60 \mathrm{~mL}$ with tap water. After imaging each culture for 5 minutes, $30 \mathrm{~mL}$ of the samples were replaced with tap water to prepare the next dilution step (1:2 dilution), followed by imaging with the DSPC. This was repeated for five dilution steps for Scenedesmus acuminatus, Oocystis solitaria, Tetraedron minimum, Botryococcus braunii and six dilution steps for Euglena sp. and Cephalodella sp. Images were then sorted out manually, whereas cropped or wrong images were not considered. The initial culture density was estimated from a subsample counted manually under an inverted microscope. 0p5x magnification. Cultures of three Daphnia species, of different size classes: D. magna, D. longispina, D. cucullata were used. They were diluted (1:2) in six decreasing levels from 200 to 7 individuals (counted manually) and imaged in a culturing flask (total volume of $60 \mathrm{~mL}$ ) for 5 minutes each. The images were manually sorted: first, all pictures containing Daphnia, including cropped or/and not in focus, were used for density estimation. Second, for the size estimation, only pictures of Daphnia in focus and not cropped were considered.

\subsection{Field deployment and comparison with traditional monitoring}

\subsubsection{Automated deployment and monitoring station}

The dual-magnification DSPC was installed at our automated monitoring station located in Greifensee, a peri-alpine, eutrophic lake in Switzerland $\left(47.35^{\circ} \mathrm{N}, 8.68^{\circ} \mathrm{E}\right)$. Specific details about the automated monitoring platform can be found in (Pomati et al., 2011). The station features a multiparametric probe Ocean7 and an automated profiler (https://www.idronaut.it/), a meteorological station (WS700-UMB from OTT Hydrometrie / LUFFT), a local computer and data network by 4G modem allowing data transmission. All data are streamlined and published in real-time on our website www.aquascope.ch.

\subsubsection{DSPC settings and data}

The DSPC is permanently installed at a depth of $3 \mathrm{~m}$, monitoring the plankton community hourly, for 10 minutes at the start of every hour, at an imaging rate of 1 frame / second. The weekly maintenance consisted of cleaning the viewports, which did not require uninstalling the DSPC and bringing it to the laboratory. The DSPC never experienced any major structural damage, and the yearly maintenance was performed only for replacing an anode, cleaning, and checking internal humidity. No interruption of function was caused by the failure of hardware. The longest interruption was due to the use of the DSPC during an international exposition in January 2019, which required uninstalling it from the monitoring station for a month.

Phytoplankton: For comparisons with traditional monitoring, we used only the 5p0x magnification and selected the images of the DSPC that were taken in the same hour as a microscopy sample. This was mostly between 10:00-12:00 in the morning. The whole 10 minutes of running time and associated images were used. The images were then classified taxonomically to the lowest possible classification level. Zooplankton: We used only the 0p5x magnification data and aggregated discrete hourly samples over an entire day to allow for comparisons with traditional twin-net tows. Specifically, we took the mean of a $24 \mathrm{~h}$ running time, 10 minutes every hour. This was to increase DSPC sample size, since net-tows concentrate hundreds of liters of water. The images of $0 \mathrm{p} 5 \mathrm{x}$ magnification were subset to every 6 seconds in order to exclude multiple imaging of the same object, since some individuals were observed to remain in the focal plane of the camera for some seconds, particularly when the water was calm. The images were then classified at the lowest possible taxonomic level using the trained CNN described in Section 2.2.

\subsubsection{Traditional sampling and microscopy}

Water samples were taken every week for 12 consecutive months starting in April 2019, except for winter months (November - early March 2020) when we sampled fortnightly. All samples were taken between 10:00 and 12:00 in the morning. Phytoplankton: Water samples were taken near the DSPC, at $3 \mathrm{~m}$ depth, with a $5 \mathrm{~L}$ Niskin bottle. An aliquot of $50 \mathrm{~mL}$ was fixed with Lugol's iodine solution and then identified and counted in the laboratory under an inverted microscope (Utermöhl method). Zooplankton: Integrated water column samples, starting from March 2020 onwards, were taken with a $95 \mu \mathrm{m}$ twin net, from $17 \mathrm{~m}$ to the surface. Samples were filtered with a $95 \mu \mathrm{m}$ mesh and fixed in $50 \mathrm{~mL}$ of $100 \%$ ethanol. Identification and counting were done under a stereomicroscope.

\subsection{Data analysis}

All data analyses and plotting were performed in the R programming environment (https://www.r-project.org/). All the laboratory and monitoring data from this study are available at https://doi.org/ 10.25678/0004BW.

\subsubsection{Laboratory calibration}

Body size: We calculated the size of objects as 2-dimensional areas of the object mask, as automatically provided by our image processing scripts for DSPC, or manually per each taxon in microscopy. We used a least-squares linear regression to correlate microscopy measurements (the benchmark) and measures taken by the DSPC, in $\log _{10}$ space. Sizedensity curves were done by using geom_density from the ggplot2 package on untransformed areas (geom_density, ggplot2 3.2.1). Density: Measurements were $\log _{10}$ transformed, with zeros set to an arbitrarily low value $(0.1)$ before the transformation. When dilution series were run multiple times, we took the mean between technical repeats. Density estimates by microscopy (the benchmark) and DSPC were then compared using linear regression for each taxon. Similarly, an overall relationship between microscopy and DSPC was calculated with linear regression in $\log _{10}$ space for each magnification.

\subsubsection{Field application}

Seasonal patterns: We estimated the mean size of the phytoplankton community (as the area of ROIs detected by the 5p0x magnification), and its densities, using the DSPC data from 2018. As a comparison to DSPC density estimates, we used the Chlorophyll-a (Chla) concentration recorded by the CTD-probe fluorimeter (https://chelsea.co.uk/products/trilux/), using averages of Chl-a measurements from 2.9 to $3.1 \mathrm{~m}$ from each profile. We classified objects at $0 \mathrm{p} 5 \mathrm{x}$ 
magnification, including zooplankton taxa and several large colonial phytoplankton, with the trained CNN using images sampled from the full dataset every 6 seconds, again to avoid multiple images of the same object. Counts were then aggregated into coarser taxonomic levels (copepods, daphnids, rotifers, predators and nauplia). Day-night patterns: To study phytoplankton daily division (cells grow during the day and divide during the night) and the consequent change in community average size, and zooplankton community day-night migrations, we used phyto- and zooplankton body size (area) and abundance (ROI / second) data over $24 \mathrm{~h}$ across 34 non-consecutive days extracted with a weekly interval from the data collected in 2020. Again, $0 \mathrm{p} 5 \mathrm{x}$ data were down sampled to one frame every 6 seconds, then ROIs were classified with the $\mathrm{CNN}$ model and finally aggregated into coarse taxonomic groups. Community diversity: Community diversity metrics were estimated using ROI counts performed manually (classification and counting of images) for the $5 \mathrm{p} 0 \mathrm{x}$ magnification, and automatically (CNN) for the 0 p5x magnification. For both comparisons, the number of data points used amounted to the weekly samples taken for traditional plankton monitoring methods $(n=44$ for phytoplankton and 30 for zooplankton). Richness, Shannon diversity and Pilou's evenness were calculated using functions of the R package vegan (2.5-6) and linear density data. Relationships between traditional monitoring and DSPC in terms of plankton abundance and diversity were estimated using leastsquares linear regression.

\section{Results}

\subsection{Laboratory calibration}

To assess the DSPC sensitivity in automatically detecting objects, how such detection scales with plankton densities, and its accuracy in extracting object features such as plankton body size (i.e. the area of the object in two dimensions), we performed laboratory experiments to compare DSPC results with traditional (manual) microscopy methods. We used living phytoplankton cultures, protists cultures, daphnia cultures and field samples for rotifers, copepods and other zooplankton species. Selected species represented a wide range of sizes, from Tetraedron minimum $\left(0.00018 \mathrm{~mm}^{2}\right)$ to Daphnia magna $\left(3.2 \mathrm{~mm}^{2}\right)$.

Overall, we found a strong linear relationship in Log-Log space $\left.\log _{10}, \mathrm{R}^{2}=0.959\right)$ between inverted microscopy (area calculation based on ImageJ) versus automated imaging and ROI area extraction using the DSPC (Fig. 2). The relationship indicates that size estimates tended to be larger in the DSPC. For both magnifications, we observed a stronger deviation from the linear relationship at the smaller size end. Fig. 2A shows how the lower resolution for smaller taxa in DSPC images makes small objects appear overpixeld and blurry, biasing the
A

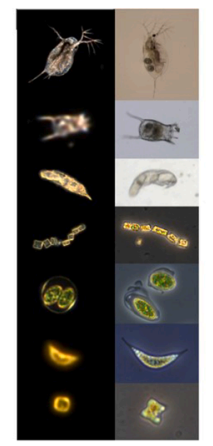

B

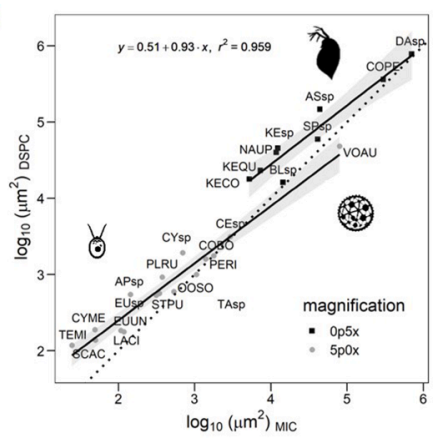

Fig. 2. Relationship between body size estimates by inverted microscopy and the DSPC. [A] Selected pictures of species by DSPC (left) and inverted microscopy (right), sorted based on size from the biggest taxon (on top) to the smallest (bottom). [B] Mean body size (ROI area) between inverted microscopy (MIC) and the plankton camera (DSPC). Dotted line $=1 / 1$ relationship, solid lines $=$ linear regression estimates $(\mathrm{p}<0.05)$ and $95 \%$ confidence intervals. automated edge detection and therefore area estimation. The only taxon lying below the 1:1 line was Volvox aureus, a very large phytoplankton colony for which we detected many partial images. Individual size distributions for each taxon confirmed an overestimation of size (as ROI area) at the lower size end. In both magnifications, density distributions of size estimates overlapped better for larger taxa, i.e. Daphnia, Cephalodella, Oocystis, compared to smaller taxa, i.e. Keratella quadrata, Scenedesmus acuminatus, Tetraedron minimum (Supplemental Fig. S1).

For all taxa, density estimations by microscopy scaled significantly and accurately with ROI/s detected by the DSPC (Fig. $3-\mathrm{R}^{2}=0.89$ and $\mathrm{R}^{2}=0.73$ for phytoplankton and zooplankton, respectively). We compared the number of objects detected per second (i.e. per frame) in the DSPC to density measurements obtained by inverted microscopy for small (5p0x) and stereomicroscopy for large (0p5x) plankton organisms. We performed serial dilutions of zoo- and phytoplankton taxa ranging of different sizes $\left(118 \mathrm{um}^{2}-3.2 \mathrm{~mm}^{2}\right)$. The overall scaling was more consistent across taxa for $5 \mathrm{p} 0 \mathrm{x}$ magnification than for $0 \mathrm{p} 5 \mathrm{x}$. However, the strength of the relationship did not depend on size.

\subsection{Field deployment and biodiversity dynamics}

To explore the potential of the new in situ imaging approach for automated monitoring of plankton community dynamics, we installed a dual-magnification DSPC permanently, at the stationary depth of $3 \mathrm{~m}$, at the Eawag platform in the middle of Lake Greifensee (Switzerland) (www.aquascope.ch). The instrument has been operating autonomously, with rare interruptions of a few days a year for maintenance, since April 2018. During this time, we have acquired imaging data to describe the dynamics of the planktonic food web at high temporal frequency, and regularly taken samples with traditional monitoring
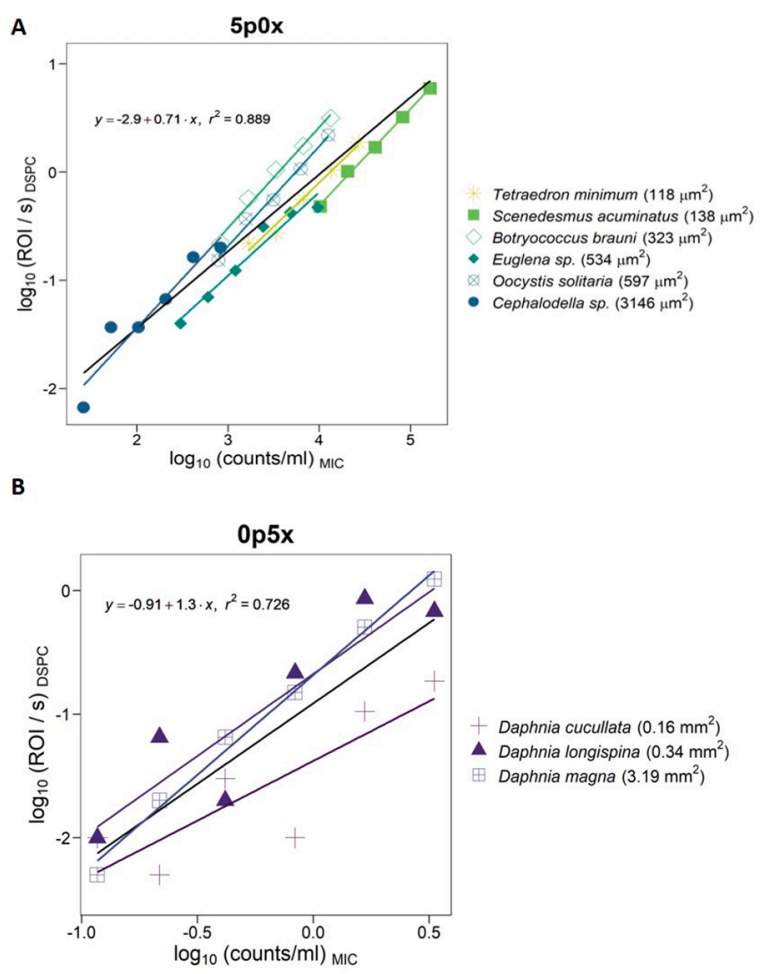

Fig. 3. Calibration of abundance estimates by DSPC relative to microscopic counts. [A] Phytoplankton and small protists are ordered according to body size (area, measured by DSPC). [B] Three different species of Daphnia were ordered according to body size (area, measured by DSPC). Black lines show the overall relationship between $\log _{10}$ transformed abundance measures by the DSPC (ROI / s) to density estimates based on inverted microscopy (counts / $\mathrm{mL}$ ). Colored lines and dots represent the regression lines for the single taxa. All relationships are significant to $\mathrm{p}<0.05$. 
approaches to compare biodiversity measurements by the DSPC to standard sampling and analysis methods. Patterns collected between 2018-2020 highlight seasonal successions and bloom dynamics, circadian rhythms in abundance and size. Comparisons with traditional methods reveal the advantages and limitations of this new approach.

\subsubsection{DSPC characterization of plankton diversity}

In over two years of data collection in Lake Greifensee we could identify 28 plankton taxa with the $0 \mathrm{p} 5 \mathrm{x}$ magnification (mostly zooplankton) and 80 taxa with $5 \mathrm{p} 0 \mathrm{x}$ magnification (mostly phytoplankton). Some taxa were present in both magnifications, i.e. ciliates, rotifers and large phytoplankton colonies. Fig. 4A displays a collage of images representing the diversity of plankton as was observed with the dual-magnification DSPC in the field, which encompassed primary producers, mixotrophs, herbivore grazers and carnivore zooplankton. Qualitatively, the high resolution and dark-field background of images
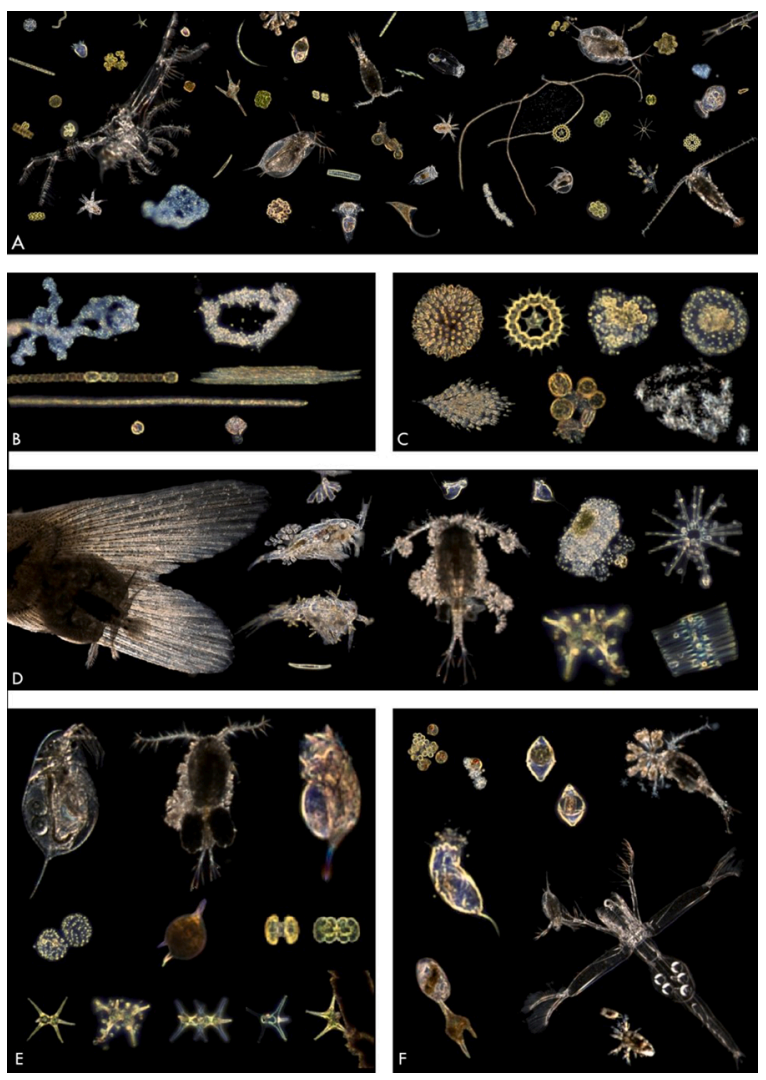

Fig. 4. DSPC images of plankton taxa and natural taxa aggregates from Lake Greifensee (pictures are not to scale). [A] Collage of different images describing the large range of taxa and forms that can be imaged with the dualmagnification DSPC. [B] Potentially toxic cyanobacteria, left to right, top to bottom: two colonies of Microcystis sp., Dolichospermum sp., Aphanizomenon flosaquae and Planktothrix rubescens. [C] Colonial algae - left to right, top to bottom: Uroglena sp., Pediastrum sp, two colonies of Coelosphaerium, (bottom row) Dinobryon sp., natural aggregates of centric diatoms and aggregates of Asterionella $s p$. [D] Hosts and parasites (or epibionts) - left to right, top to bottom: parasite on a fish fin, Epistylis sp.on copepods, chytrid fungi on Asterionella formosa and Fragilaria crotonensis colonies, and unknown epibionts on Staurastrum sp. [E] Reproduction and life stages from left to right, top to bottom: Daphnia sp., Cyclops sp. and Keratella cochlearis (with eggs), Coelosphaerium sp., a cyst of Ceratium hirundinella, two dividing stages of Cosmarium sp., two Staurastrum sp. - a dividing one and a dying individual. [F] Individual trophic interactions - from left to right, top to bottom: dinoflagellates eating green algae, two Peridinium sp. after eating centric diatoms, Cyclops sp. Eating a rotifer colony, a rotifer eating algal aggregate, Leptodora kindtii hunting for a Daphnia, Ceratium hirundinella interacting with a ciliate and a copepod nauplius interacting with an unknown organism. made taxon identification possible and facilitated image processing and training of a deep-learning classifier for zooplankton (see further Sections), with both morphology and color playing an important role.

The DSPC imaging approach represented a non-invasive and unbiased method to study plankton in the field, since taxa and their microenvironment were monitored without disturbing natural behavior, heterospecific aggregates and colonies structures (Fig. 4B-D). Some potentially toxic algae, such as the cyanobacteria Mycrocystis or Planktothrix, were successfully imaged and clearly recognized (Fig. 4B). Apart from colonies and natural aggregates (such as lake snow - Fig. 4C), we observed direct interactions between, for example, hosts and parasites (Fig. 4D) or predators and prey (Fig. 4F). Some of these images might be the first-ever to capture direct individual trophic interactions in freshwater microbes from the field, such as ciliates and rotifers preying on phytoplankton (Fig. 4F). Colored images enabled us to distinguish between living and dead cells, and to observe plankton division and reproduction, i.e. by imaging dividing cells or individuals carrying eggs (Fig. 4E). For some large zooplankton like Daphnia, it is possible to count eggs from images. The images also allowed us to identify and count different life stages of zooplankton, as is the case for juvenile copepods and nauplii.

\subsubsection{Comparison with traditional sampling and microscopy}

We compared phytoplankton and zooplankton biodiversity metrics estimated by the DSPC at $3 \mathrm{~m}$ to those obtained by traditional monitoring methods. Our regular monitoring campaign in Lake Greifensee with manual sampling and microscopy captured in total 159 different zoo- and phytoplankton taxa (Supplemental Table S5). We could observe $88.7 \%$ (141) of them in the DSPC. We noticed that issues in identification arise when relatively small or thin taxa are not imaged, i.e Chromulina sp. or Leptothrix sp. From the DSPC images, we could create 101 unique and distinguishable zoo- or phytoplankton taxa categories. Of them, 95 were identified at least to genus level, which is about $30 \%$ less than what can be archived with traditional microscopy (i.e a total of 134 taxa identified at genus level). The taxonomic identification in DSPC images was variable depending on the taxon and on whether the characters that are used for classification were distinguishable from the images. For both magnifications, taxonomic classification was harder at the lower end of the detection spectrum of the cameras. Phytoplankton richness estimates by the two approaches were in overall agreement, however considering the differences in both sampling and analysis methods, the DSPC data systematically underestimates richness compared to traditional methods (Fig. 5A). Temporal trends in phytoplankton richness highlighted this offset, which varied over time, depending on phytoplankton community composition: the data showed better agreement between the two approaches in winter, when fewer and easily detectable taxa, such as Cryptomonas $s p$., were present (Fig. 5B). Zooplankton communities are in general less rich than phytoplankton, and both approaches detected low diversity in our samples (max. 11 species). We did not find a significant correlation between zooplankton richness estimates by DSPC and traditional methods, and the relationship appeared even negative (Fig. 5C). In the case of zooplankton richness estimation, the DSPC, which averaged data over a $24 \mathrm{~h}$ period, detected higher richness than the traditional method based on a daytime net-tow and microscopy.

Total phytoplankton and zooplankton community densities estimated by the DSPC agreed well with data from traditional sampling methods (Fig. 6A-B). The phytoplankton relationship was statistically significant and robust: ROI/s generated by the DSPC correlated strongly also with Chl-a concentrations measured in situ next to the instrument (Fig. 5 and Supplemental Fig. S2). For zooplankton, the relationship between density estimates by daytime net-tows and DSPC $24 \mathrm{~h}$ averages were statistically significant but poor (Fig. 6B). The relationship between phytoplankton taxa relative abundances (i.e. evenness) by DSPC and traditional methods was not statistically significant and poor, with data however scattered around the $1 / 1$ line (Fig. 6C). Zooplankton taxa 
A

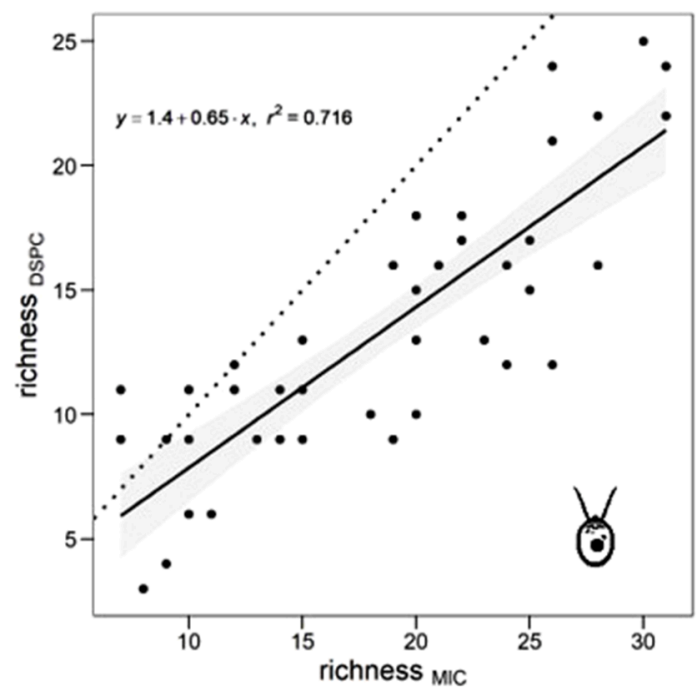

C

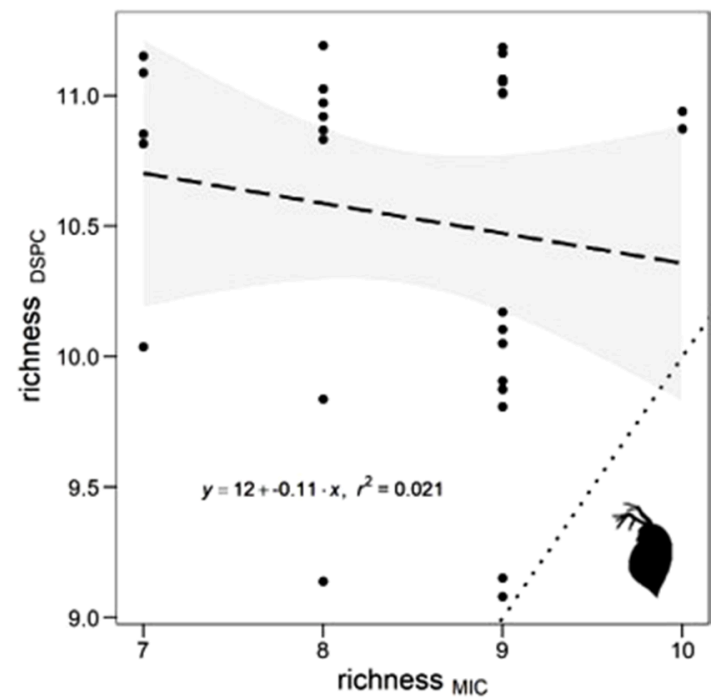

B

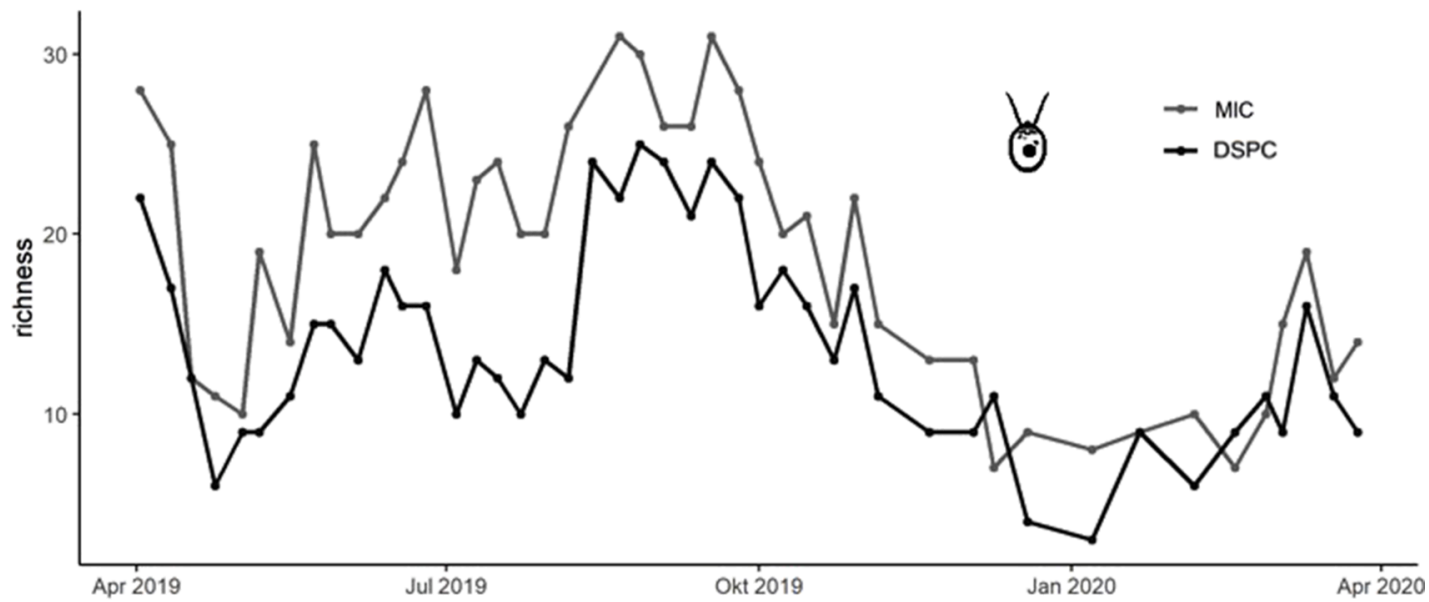

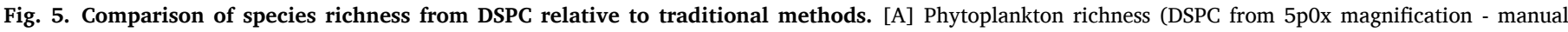

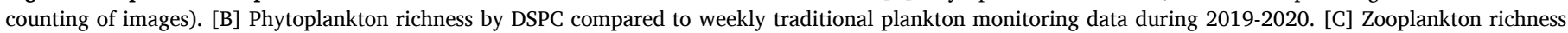

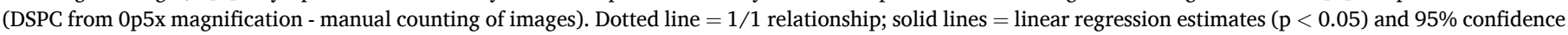
intervals; dashed lines $=$ not significant $(p>0.05)$.

evenness, instead, showed a significant and relatively strong relationship between the two approaches (Fig. 6D).

\subsubsection{Seasonal patterns}

The DSPC data in 2018 depicted the most intense algal bloom in Lake Greifensee since the early 1990s in terms of phytoplankton densities (see Chl-a levels in Fig. 7A). ROI/s collected by the DSPC for the 5p0x magnification correlated highly with the Chl-a measured by a CTD fluorometer at $3 \mathrm{~m}$ depth $\left(\mathrm{R}^{2}=0.94, p<0.001\right.$; Supplemental Fig. S2). Both Chl-a concentration and ROI/s by the DSPC (Fig. 7) showed a relatively small increase in phytoplankton at the end of July, and then a large accumulation in the middle of September, which was characterized by a relatively smaller mean phytoplankton community size (ROI area) compared to non-bloom periods (Fig. 7A). The late summer bloom was dominated by the small single-cell taxon Oocystis sp., as identified by DSPC images, while pre-bloom communities were more heterogeneous (Supplemental Fig. S3). Patterns of co-occurring zooplankton taxa and their abundance (ROI/s) showed that relatively high density of herbivore grazers (particularly daphnids) in the pre-bloom phase (middle of August) was followed by a decrease in their abundance after the DSPC detected the presence of carnivore species (particularly Leptodora) at the onset of the phytoplankton bloom (Fig. 7B). This release from top-down grazing pressure might have been involved in the emergence of the bloom, which was dominated by a small edible taxon. At the end of September, the algal bloom declined as we observed an increase in small fast-growing herbivores (i.e. rotifers), which might have been involved in bloom termination (Fig. 7B).

\subsubsection{Diel patterns}

Time series generated by the DSPC, which was set for $10 \mathrm{~min}$ of measurements every hour, 24/7, allowed the study of diel size dynamics and of zooplankton day/night migratory behavior (Fig. 8). Phytoplankton mean community size showed to be slightly larger during the day compared to the night (when both grazing and cell division mostly occur) (Fig. 8A). Zooplankton mean community size was larger, instead, during the night than during the day (Fig. 8B). This was likely due to different migration behaviors of large relative to small herbivore grazers (Fig. 8C-F). Copepods and daphnids were more abundant (higher ROI/s) during the night, and peaked between midnight and 3 am (Fig. 8C-D). During the day, daphnids were almost undetectable. Conversely, nauplii 
A
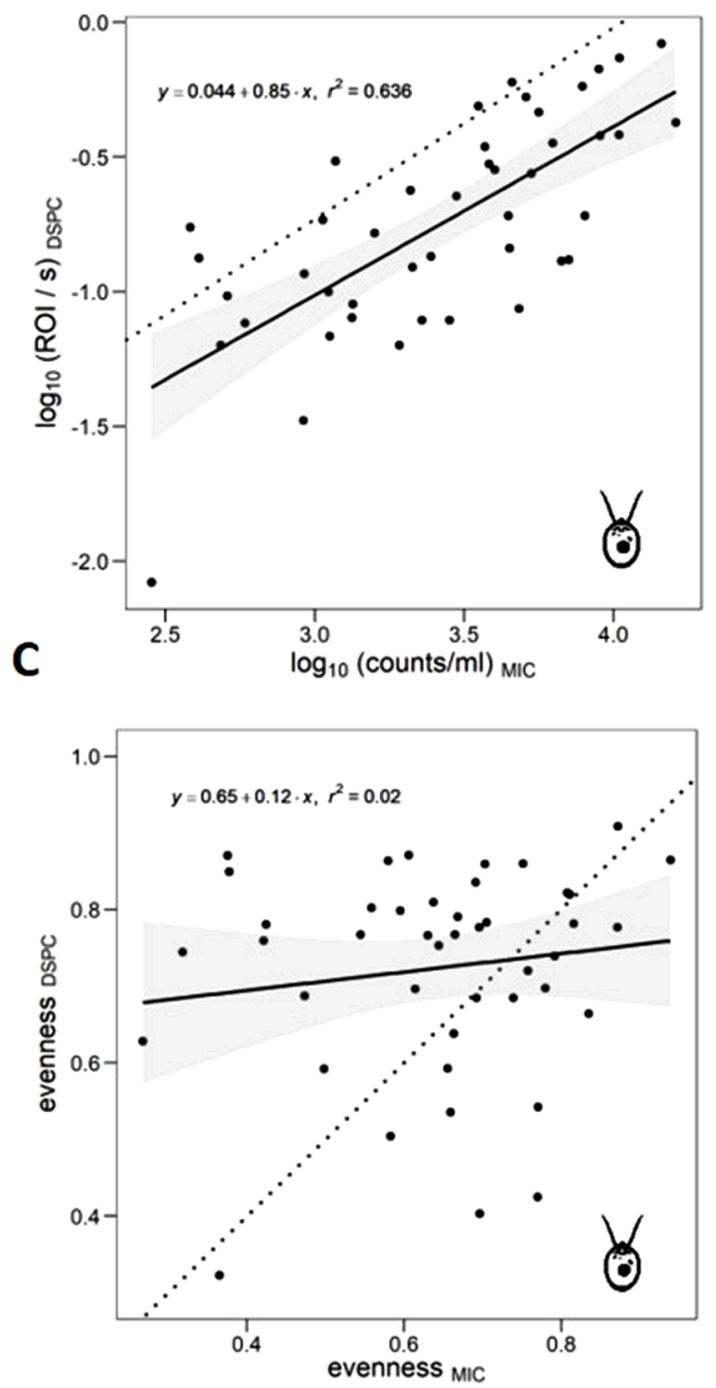

B
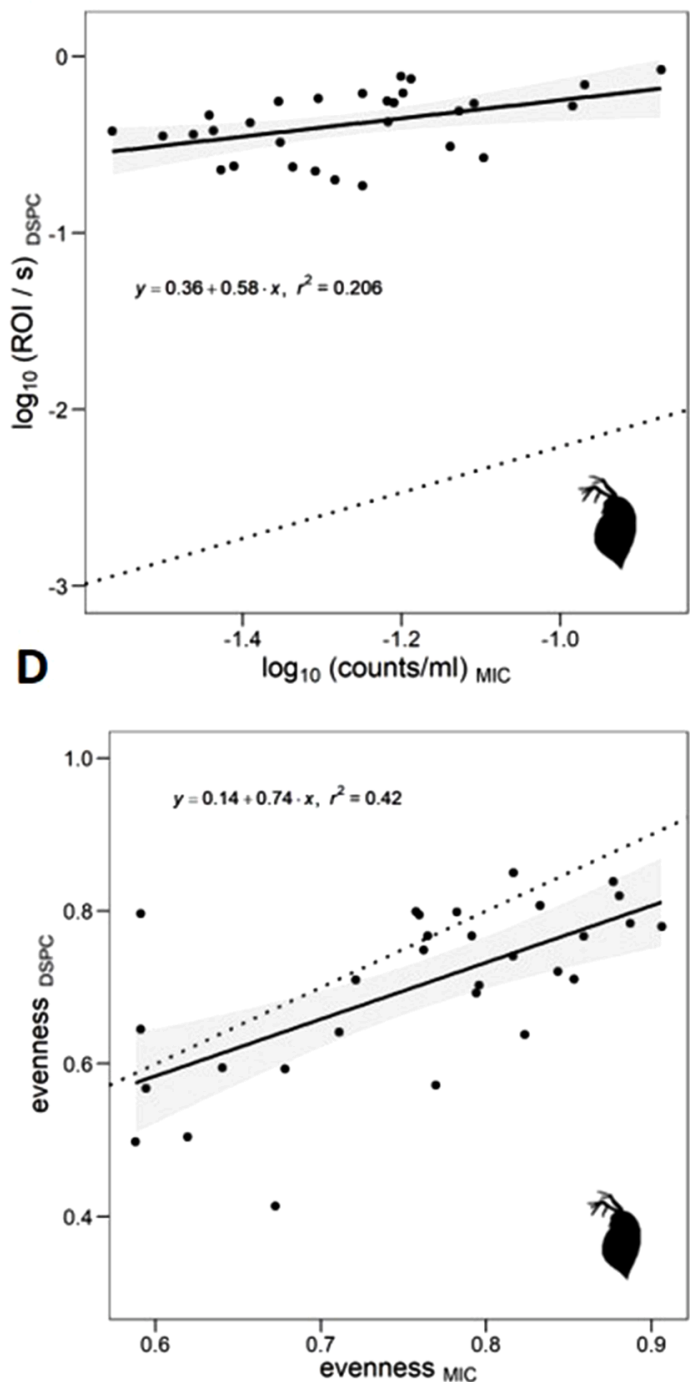

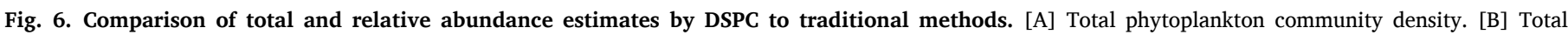

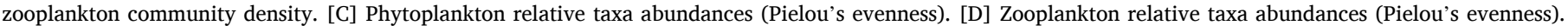

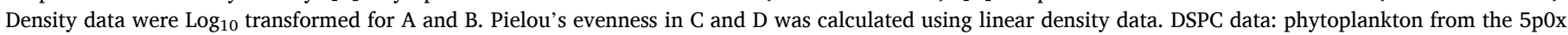

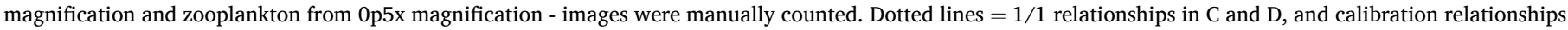
from laboratory serial dilutions in A and B (Fig. 3), solid lines = linear regression estimates $(p<0.05)$ and $95 \%$ confidence intervals.

and rotifers (the most abundant group at $3 \mathrm{~m}$ ) did not show any significant circadian patterns (Fig. 8E-F).

\section{Discussion}

The DSPC allowed imaging plankton individuals, colonies, and aggregates of taxa and other suspended solids (e.g. lake snow) without disrupting natural arrangements of interacting organisms, their microenvironment, or their behavior. The instrument reliably delivered high-resolution images in real-time, and measurements of traits like size, shape and color. This included detection capability across a widereaching size range of the planktonic food web, capturing around $90 \%$ of the plankton taxa in Lake Greifensee when compared to traditional monitoring. Plankton taxa found in Lake Greifensee, including for example herbivore grazer (i.e. copepods and daphnids) or toxic cyanobacteria (i.e. microcystis $s p$. and planktothrix rubescens), are common members of plankton communities in all temperate lakes - suggesting that the DSPC could be effective across other lakes and reservoirs. Detecting particles from nanoplankton to mesoplankton with high resolution is a dynamic range never achieved before in other in-situ imaging systems (Lombard et al., 2019). Taxonomic identification was variable depending on the detected taxon and the resolution of the image. In general, the colored images with dark backgrounds afforded high object definition and contrast, qualities that facilitate the identification of taxa and the annotation of ROIs for the training of deep-learning models. Automated image classification was achieved for plankton with the 0p5x magnification in this study (Supplemental methods) and elsewhere (Campbell et al., 2020; Orenstein et al., 2020). The training of a phytoplankton classifier will be manageable with relatively low effort (Kenitz et al., 2020), however more tedious due to the larger number of taxa to annotate. An approach based on imaging has the potential to yield high frequency and standardized plankton monitoring data (Lombard et al., 2019) and, boosted by continuous (and open-source) advances in image processing and artificial intelligence, offers the prospect to automate and standardize the taxonomic classification of plankton (MacLeod et al., 2010).

Time series collected by the stationary DSPC in Lake Greifensee depicted seasonal succession patterns, bloom dynamics and circadian 


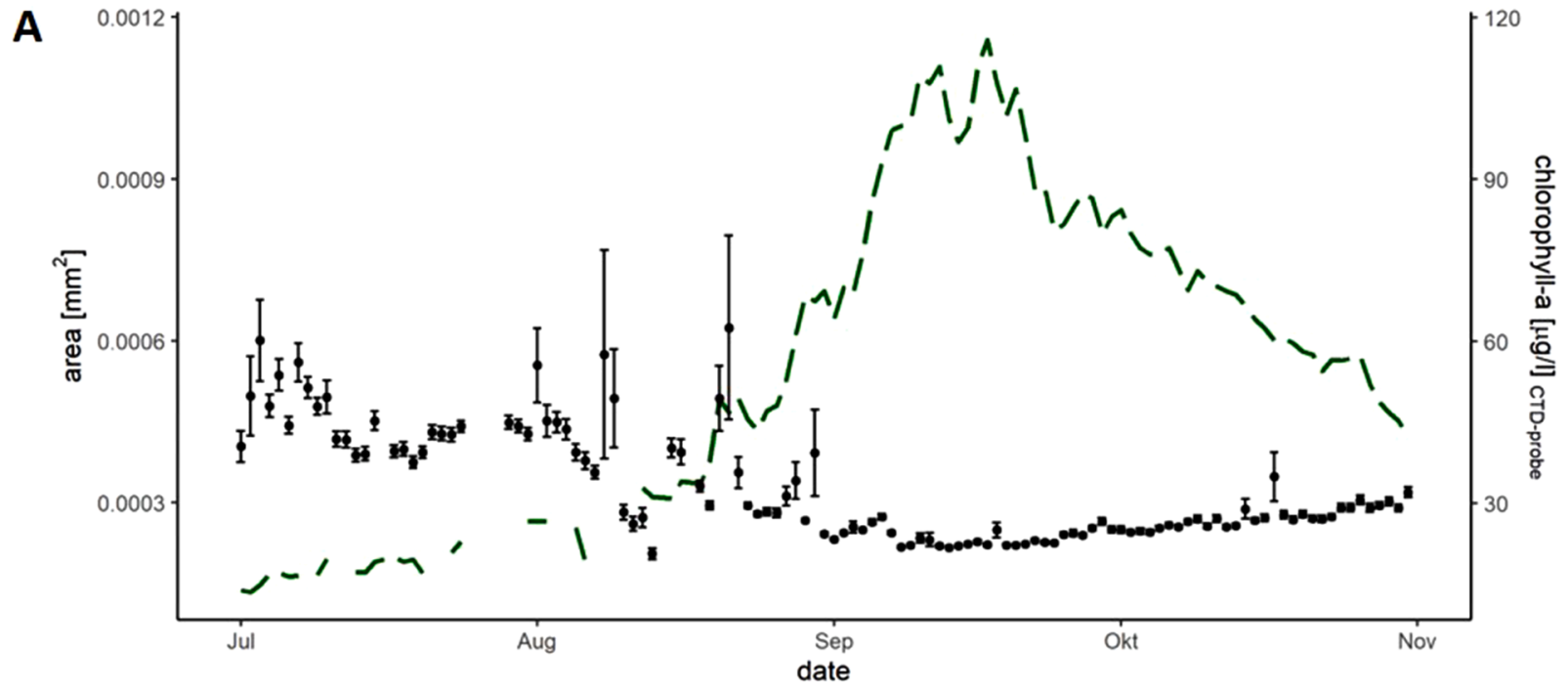

B

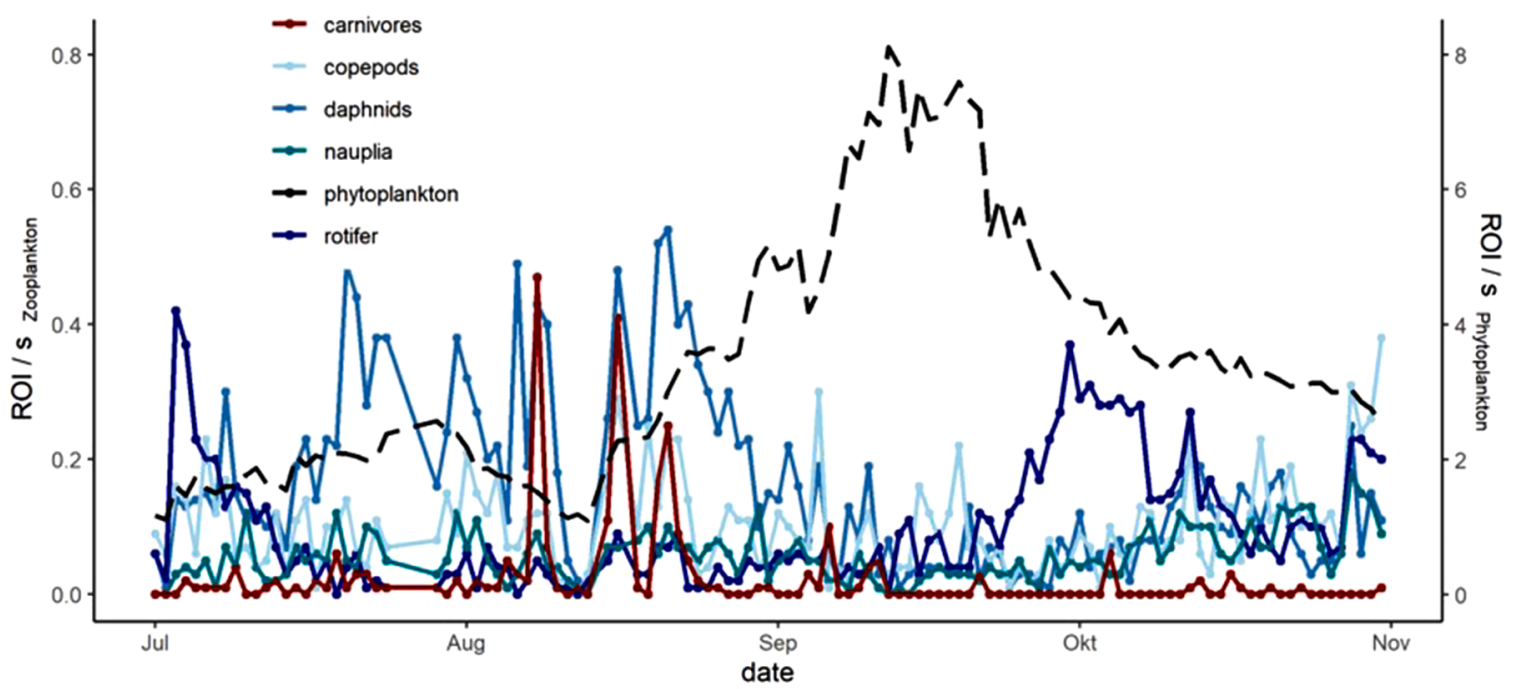

Fig. 7. Patterns from DSPC monitoring data from Lake Greifensee (2018). [A] Chl-a data from the CTD and fluorimeter (dashed green line), and phytoplankton community size by DSPC (points represent the mean ROI size in the 5p0x magnification $+/$ - standard error of the mean). [B] Patterns in plankton density (ROI/s) dynamics from the DSPC: colored lines represent different zooplankton taxa (based on CNN automated classification, from 0p5x magnification, see Supplemental Table S3), dashed black line represents phytoplankton (from the 5p0x magnification). Data represent one DSPC measurement per day (10 min running time, subset to 6 seconds for zooplankton) taken during the night (01:00).

fluctuations with a temporal and spatial (i.e. microenvironment and morphology) resolution that would have been virtually impossible to collect based on traditional methods. In particular, the DSPC data included all essential planktonic groups to understand and forecast community dynamics (including algal blooms), but are often overlooked in routine monitoring (e.g. ciliates, chytrid fungi) (Fig. 2). In addition, automated underwater imaging allows following the dynamics of important individual-level traits distributions such as size, shape, and pigmentation, which are extremely hard to measure manually on large numbers of individuals. Morphological characteristics of phytoplankton (e.g. length, surface, coloniality, and pigmentation) correlate with species functional properties (e.g. resource acquisition, growth rate, sinking rate, grazing resistance), and therefore with population abundances and responses to environmental gradients (Fontana et al., 2019; Kruk et al., 2010; Pomati et al., 2013).

In this study, DSPC imaging and feature extraction allowed calculation of bi-dimensional areas for ROIs, which related well to areas of plankton taxa based on traditional microscopy and manual measurements. Although measured in two dimensions, the body size estimates by one or two linear dimensions scale with biovolume estimates by traditional methods (Mittler et al., 2019). Body size was overestimated by the DSPC at the lower size end, where both regression lines for the 0p5x and 5p0x magnification deviated for smaller species from a perfect $1 / 1$ relationship. The deviation was due to a lack of resolution in pixels for smaller individuals, particularly when slightly out-of-focus. This error can be corrected using the relationship derived by laboratory calibration (Fig. 2). The overestimation of size would be even greater when using estimates of 3-D biovolume (rather than 2-D area), and care should be given when calculating biovolume estimates.

Similarly, DSPC measures of plankton densities (ROI / s, or alternatively ROI / frame), can be translated into a number of individuals per unit of volume (e.g. L) based on the laboratory calibration (Fig. 3). Note, however, that the initial densities in our laboratory experiments were much higher than those usually found in nature: for the goal of an $a b$ solute quantitation of plankton densities, we suggest a laboratory calibration targeted towards specific instrument settings and realistic 
A

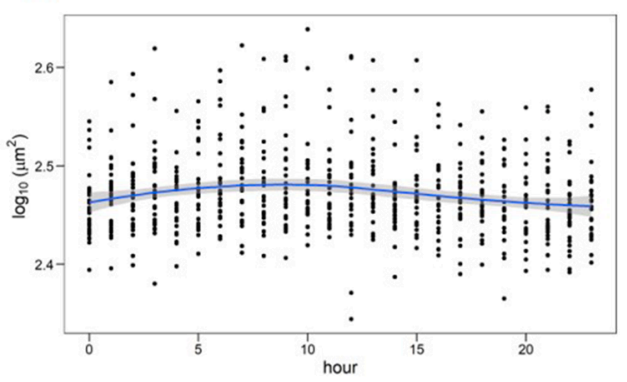

C

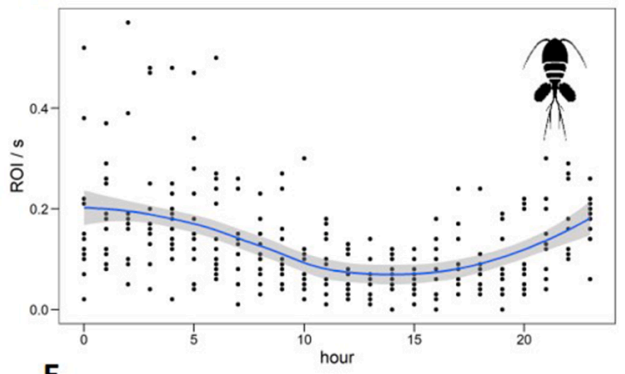

E

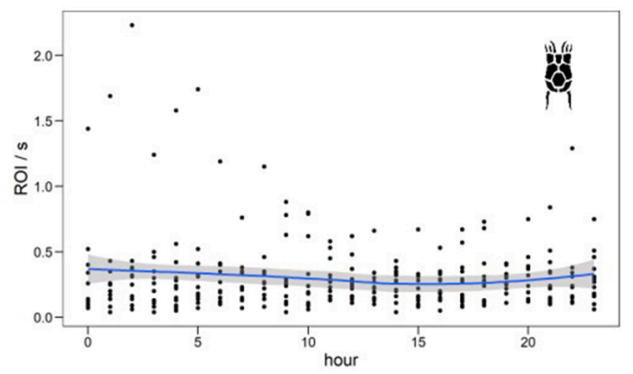

B

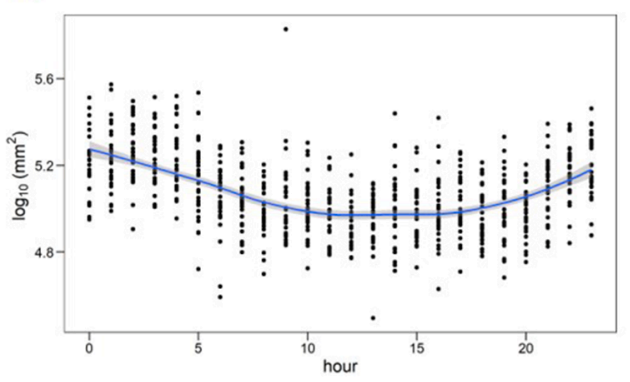

D

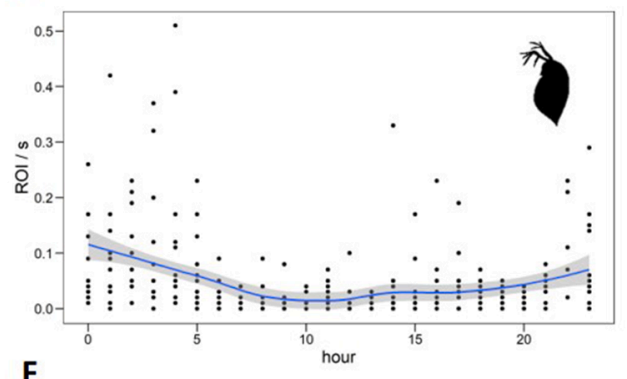

$\mathbf{F}$

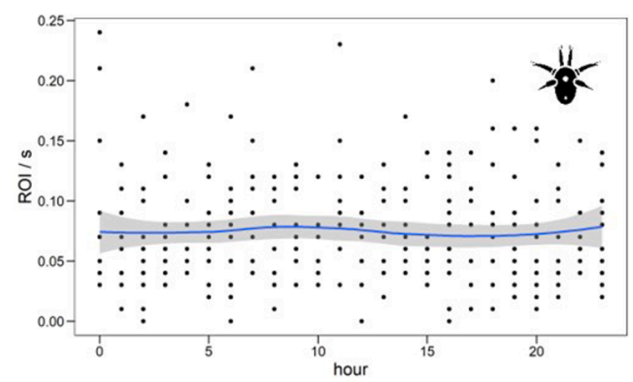

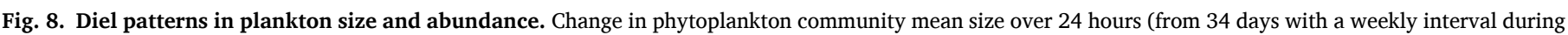

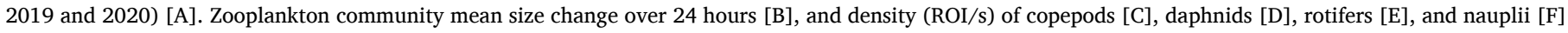
(from 34 random days in 2020). Zooplankton counts are based on CNN automated classification, from 0p5x magnification, see Supplemental Table S3.

densities. Measuring densities based on detected ROI / s would be suitable to estimate relative abundances. Other issues emerging from the comparison of DSPC data with traditional plankton sampling include i) the difference between methods in terms of sampled volume that is used to estimate plankton community indices; ii) no detection of phytoplankton $<10 \mu \mathrm{m}$, resulting in an underestimation of some small but common species; iii) the spatial heterogeneity and circadian behavior of zooplankton.

The DSPC imaged volume (Supplemental Table S1) is small compared to traditional monitoring samples, and variable depending on water optical conditions (Orenstein et al., 2020). Unlike traditional counting methods, the DSPC cannot easily change sampled volume to respond to changes in densities. While we can increase imaging sampling effort by applying a space for time substitution, assessing the actual differences in sample size between DSPC and traditional methods remains difficult. This disagreement in sampled volume, which applies to almost all automated in-situ methods, has been already acknowledged (Lombard et al., 2019). While automated imaging data allow the scaling of measures of diversity based on sample size (e.g. by temporally aggregating data and applying rarefaction and probability estimate of metrics) (Chase and Knight, 2013), this is generally impossible with traditional sampling methods. Those differences in scaling hamper our understanding of why imaging and traditional methods sometimes fail to converge to the same diversity estimates (Fig. 5-6).

The DSPC showed difficulties in detecting small species for the $5 \mathrm{p} 0 \mathrm{x}$ magnification, and in quantifying densities of large rare species, including carnivorous zooplankton, for the $0 \mathrm{p} 5 \mathrm{x}$ magnification. The lack of taxonomic resolution for small phytoplankton taxa (in $5 \mathrm{p} 0 \mathrm{x}$ ) is a limitation of the optical system itself, solvable by an extra (higher) magnification. The correlations between DSPC and microscopy estimates for phytoplankton richness and total abundance were however robust (Figs. 5A and 6A), with the limitations in taxonomic resolution becoming more evident in the comparison of relative abundance estimates (Fig. 6C). The lack of reliable quantitation of large zooplankton taxa in the 0p5x magnification, instead, resides in the relationship between spatial density and body size of organisms (White et al., 2007). Imaging with the DSPC for a longer time, particularly at night when these organisms are more active, might ameliorate this problem by increasing the sampling effort. Due to a relatively small focal volume, however, large individuals tend to be imaged and cropped multiple times. We suggest that large carnivorous zooplankton can only be studied with presence/absence data or during periods when their densities reach very high levels.

Zooplankton species have patchy distributions and exhibit diel vertical and horizontal migrations. Measuring their community and population properties could lead to systematic biases if based on nonreplicated or only daytime samples (Doubek et al., 2020). We propose that the poor correlation between DSPC and traditional sampling for zooplankton richness and abundances was caused by the heterogeneous distribution of taxa over space and time - further supported by high levels of variation among replicated samples, particularly for traditional methods (Fig. S7). In this perspective, DSPC data (integrated over $24 \mathrm{~h}$ 
and including day/night samples) may be more trustworthy than the traditional daytime net-tows, which have been previously suggested not to be an appropriate approach for monitoring (Doubek et al., 2020).

Availability of high-resolution real-time plankton data represents a potential breakthrough in both applied and fundamental plankton ecology and might be more important for water quality monitoring than a fine taxonomic resolution. High-frequency data have been shown to provide exciting prospects for modeling, understanding and predicting plankton dynamics and ecosystem processes (Fontana et al., 2018; Thomas et al., 2018). The seasonal patterns depicted in Fig. 7 highlight the rich description of the ecological succession of taxa that DSPC data can offer for studying plankton communities. Natural processes fostering high plankton biodiversity on one hand, and leading to the emergence of harmful algal blooms, on the other hand, represent unresolved questions in aquatic ecology (Burford et al., 2020; Fox et al., 2010; Huisman et al., 2018; Li and Chesson, 2016). The DSPC was able to detect (and count) all the most common and potentially toxic cyanobacterial taxa forming blooms in lakes, including their large colonies (Fig. 2). If supported by an automated deep-learning classifier and paired with meteorological and CTD data (and/or a calibrated lake process models), the DSPC could be instrumental to develop forecasting models for harmful cyanobacterial blooms, and the stability and biodiversity of natural plankton communities (Pomati et al., 2011; Thomas et al., 2018).

Time series generated by instruments like the DSPC can be combined with novel data analysis approaches, including equation-free modeling and machine-learning, to reverse engineer community ecology based on observational information and to develop effective forecasting tools (Deyle et al., 2016; Martin et al., 2018; Sugihara et al., 2012; Thomas et al., 2018). The combination of high-frequency data, a broad taxonomic spectrum and artificial intelligence (for data assimilation and modeling), presents an unparalleled potential for capturing the processes acting in natural ecosystems. This approach offers great opportunities to advance our understanding of the mechanisms controlling the dynamics of the complex networks of interacting plankton species.

\section{Conclusions}

The DSPC presented in this article allowed us to obtain automated plankton data at the food web level with high temporal frequency and adequate image resolution. The plankton camera employed here was demonstrated to be very robust and reliable, showed low requirements in terms of maintenance and running costs, and offered complete automation. We conclude from our comparison with traditional monitoring methods that the DSPC is mature as a research tool and suitable for stable continuous deployment, with great potential for application in water quality monitoring.

Automated in-situ imaging data are available in real-time, and they are shareable, traceable, transparent, and independently verifiable. Biases in the data processing and analysis can be corrected by simply reprocessing and re-analyzing archived raw data. Yet, new-generation monitoring tools like the DSPC present the challenge of managing and analyzing large datasets. This might prove particularly relevant if underwater imaging cameras become more affordable and, to increase sampling effort or account for spatial variability in water bodies (both vertical and horizontal), multiple instruments are employed locally and used in full-time operation (e.g. to gain power for density estimates of rare taxa). Recent publications suggest that this transition from relatively expensive tools to more affordable, and therefore scalable, instruments are already underway (Lertvilai, 2020). Streamlined and open-source data management protocols, image processing software and automated image classification algorithms are becoming more and more popular. The DSPC can be employed for both fundamental and applied ecology, allowing calibration of lake ecological models, and supporting water quality monitoring with real-time plankton data.

\section{Contributions}

E.M., T.K., M.R., C.E., P.I., S.R.D. and F.P. planned the study. T.K. and M.R. performed field sampling and taxonomic classifications. C.E. installed the instrument in Lake Greifensee and ensured constant functioning. P.R. and J.S.J. designed and built the instrument. M.B.-J., T.L. and T.H. developed and trained the CNN. N.S. and S.R.D. created a script for zooplankton appendix erosion. E.M performed the data processing and analysis. E.M., T.K., M.R., P.I., M.B.-J., S.D. and F.P. drafted the manuscript. All authors provided critical feedback and approved the final version of the manuscript.

\section{Declaration of Competing Interest}

The authors declare that they have no known competing financial interests or personal relationships that could have appeared to influence the work reported in this paper.

\section{Acknowledgments}

We thank O. Köster, M. Koss and L. De Ventura for the fruitful discussions on the advantages and limitations of the imaging approach. This research was funded by the Swiss Federal Office for the Environment (contract Nr Q392-1149) and the Swiss National Science Foundation (project 182124). F.P and P. I. also acknowledge the Eawag DF project Cyanoswiss (\#5221.00492.012.04). F.P. and M.B.-J. acknowledge the Eawag DF project Big-Data Workflow (\#5221.00492.999.01).

\section{Supplementary materials}

Supplementary material associated with this article can be found, in the online version, at doi:10.1016/j.watres.2021.117524.

\section{Appendix. Supplementary Materials and Methods}

CNN classifier for $0 \mathrm{p} 5 \mathrm{x}$ images

The model we used for classifying zooplankton objects is a neural network with two convolutional layers, with kernel size (number of filters) of respectively 24 and 12 (64 and 32) and relu activations, followed by batch normalization and max pooling modules, and a dense layer with softmax activation ${ }^{2}$. The loss function was a categorical crossentropy. We provide our code and trained model at: https://github. com/mbaityje/plankifier/releases/tag/v1.1.1

The default code settings contain all the hyperparameter choices we made. The dataset we used consisted of 17909 images (Table S2), of which $80 \%$ were used for training, and $20 \%$ for testing. The training data and further benchmarks on this model, along with more sophisticated ones which reach higher performance, will be published separately $^{3}$. The performances that we show in this section are based on further independent data.

We rely on abstention to be able to tune the precision-recall trade off in a simple manner. This consists of labeling as unclassified all the images for which the classifier has a confidence lower than a threshold $\theta$. In Fig. S7 we show the precision and recall of our model with $\theta=0$, on the classes that are relevant for our study. The average precision is 0.84 , and the average recall is 0.8 . If we set $\theta=0.8$, the average precision is 0.91 , and the average recall is 0.67 , which means that around $30 \%$ of the data

\footnotetext{
${ }^{2}$ Despite its simplicity, this model obtains satisfactory performances, and has the additional advantage of being very lightweight. This allows us to share it, already trained, in our gihtub repository github.com/mbaityje/plankifier. The consequence is that practitioners can use it directly through the simple commands provided in the release documentation.

${ }^{3}$ Kyathanahally, S., Hardemann, T.,Merz, E., Reyes, M., Kozakiewicz, T., Isles, P., Pomati, F., Baity-Jesi, M., In preparation.
} 
is not being classified, but the data that gets classified has a very low error rate. The data shown in this paper uses $\theta=0.8$.

Since in this study we were interested in a higher-level taxonomic description of the observed organisms, we created some macro-classes, each comprising more than one of the classes described in Table S3. Obviously, the classifier performances with the macro-classes are at least as good as those of Fig. S7.

\section{References}

Bartley, T.J., McCann, K.S., Bieg, C., Cazelles, K., Granados, M., Guzzo, M.M., MacDougall, A.S., Tunney, T.D., McMeans, B.C., 2019. Food web rewiring in a changing world. Nat. Ecol. Evol. 3, 345-354.

Behrenfeld, M.J., Randerson, J.T., McClain, C.R., Feldman, G.C., Los, S.O., Tucker, C.J., Falkowski, P.G., Field, C.B., Frouin, R., Esaias, W.E., Kolber, D.D., Pollack, N.H., 2001. Biospheric primary production during an ENSO transition. Science 291, 2594-2597.

Burford, M.A., Carey, C.C., Hamilton, D.P., Huisman, J., Paerl, H.W., Wood, S.A., Wulff, A., 2020. Perspective: Advancing the research agenda for improving understanding of cyanobacteria in a future of global change. Harmful Algae 91. https://doi.org/10.1016/j.hal.2019.04.004.

Campbell, R.W., Roberts, P.L., Jaffe, J., 2020. The Prince William Sound Plankton Camera: a profiling in situ observatory of plankton and particulates. ICES J. Mar. Sci. $77,1440-1455$.

Cardinale, B.J., Duffy, J.E., Gonzalez, A., Hooper, D.U., Perrings, C., Venail, P., Narwani, A., Mace, G.M., Tilman, D., Wardle, D.A., Kinzig, A.P., Daily, G.C., Loreau, M., Grace, J.B., Larigauderie, A., Srivastava, D.S., Naeem, S., 2012. Biodiversity loss and its impact on humanity. Nature 486, 59-67.

Chase, J.M., Knight, T.M., 2013. Scale-dependent effect sizes of ecological drivers on biodiversity: why standardised sampling is not enough. Ecol. Lett. 16 (1), 17-26. Suppl.

Chorus, I., Bartram, J., 1999. Toxic Cyanobacteria in Water: A Guide to their Public Health Consequences, Monitoring and Management. CRC Press.

Deyle, E.R., May, R.M., Munch, S.B., Sugihara, G., 2016. Tracking and forecasting ecosystem interactions in real time. Proc. Biol. Sci. 283 https://doi.org/10.1098/ rspb.2015.2258.

Directive, 2000. 2000/60/EC of the European Parliament and of the Council of 23 October 2000 Establishing a framework for Community action in the field of water policy. Official J. Eur. Commun. 22.

Doubek, J.P., Goldfarb, S.K., Stockwell, J.D., 2020. Should we be sampling zooplankton at night? Limnol. Oceanogr. Lett. 5, 313-321.

Falkowski, P., 2012. Ocean science: the power of plankton. Nature 483, S17-S20.

Fontana, S., Thomas, M.K., Moldoveanu, M., Spaak, P., Pomati, F., 2018. Individual-level trait diversity predicts phytoplankton community properties better than species richness or evenness. ISME J. 12, 356-366.

Fontana, S., Thomas, M.K., Reyes, M., Pomati, F., 2019. Light limitation increases multidimensional trait evenness in phytoplankton populations. ISME J 13, $1159-1167$.

Fox, J.W., Nelson, W.A., McCauley, E., 2010. Coexistence mechanisms and the paradox of the plankton: quantifying selection from noisy data. Ecology 91, 1774-1786.

Guillard, R.R.L., 1975. Culture of phytoplankton for feeding marine invertebrates. In: Smith, W.L., Chanley, M.H. (Eds.), Culture of Marine Invertebrate Animals: Proceedings - 1st Conference on Culture of Marine Invertebrate Animals Greenport. Springer US, Boston, MA, pp. 29-60.

Ho, J.C., Michalak, A.M., Pahlevan, N., 2019. Widespread global increase in intense lake phytoplankton blooms since the 1980s. Nature 574, 667-670.

Huisman, J., Codd, G.A., Paerl, H.W., Ibelings, B.W., Verspagen, J.M.H., Visser, P.M., 2018. Cyanobacterial blooms. Nat. Rev. Microbiol. 16, 471-483.
Hunter-Cevera, K.R., Neubert, M.G., Olson, R.J., Solow, A.R., Shalapyonok, A., Sosik, H. M., 2016. Physiological and ecological drivers of early spring blooms of a coastal phytoplankter. Science 354, 326-329.

Johnson, Z.I., Martiny, A.C., 2015. Techniques for quantifying phytoplankton biodiversity. Ann. Rev. Mar. Sci. 7, 299-324.

Kenitz, K.M., Orenstein, E.C., Roberts, P.L.D., Franks, P.J.S., Jaffe, J.S., Carter, M.L., Barton, A.D., 2020. Environmental drivers of population variability in colonyforming marine diatoms. Limnol. Oceanogr.

Kruk, C., Huszar, V.L.M., Peeters, E.T.H.M., Bonilla, S., Costa, L., Lürling, M. Reynolds, C.S., Scheffer, M., 2010. A morphological classification capturing functional variation in phytoplankton. Freshw. Biol. 55, 614-627.

Lertvilai, P., 2020. The In situ Plankton Assemblage eXplorer (IPAX): an inexpensive underwater imaging system for zooplankton study. Methods Ecol. Evol. 11, 1042-1048.

Li, L., Chesson, P., 2016. The effects of dynamical rates on species coexistence in a variable environment: the paradox of the plankton revisited. Am. Nat. 188, E46-E58.

Lombard, F., Boss, E., Waite, A.M., Vogt, M., Uitz, J., Stemmann, L., Sosik, H.M., Schulz, J., Romagnan, J.-B., Picheral, M., Pearlman, J., Ohman, M.D., Niehoff, B., Möller, K.O., Miloslavich, P., Lara-Lpez, A., Kudela, R., Lopes, R.M., Kiko, R., KarpBoss, L., Jaffe, J.S., Iversen, M.H., Irisson, J.-O., Fennel, K., Hauss, H., Guidi, L. Gorsky, G., Giering, S.L.C., Gaube, P., Gallager, S., Dubelaar, G., Cowen, R.K., Carlotti, F., Briseño-Avena, C., Berline, L., Benoit-Bird, K., Bax, N., Batten, S., Ayata, S.D., Artigas, L.F., Appeltans, W., 2019. Globally consistent quantitative observations of planktonic ecosystems. Front. Marine Sci. 6, 196.

MacLeod, N., Benfield, M., Culverhouse, P., 2010. Time to automate identification. Nature 467, 154-155.

Martin, B.T., Munch, S.B., Hein, A.M., 2018. Reverse-engineering ecological theory from data. Proc. Biol. Sci. 285 https://doi.org/10.1098/rspb.2018.0422.

Mittler, U., Blasius, B., Gaedke, U., Ryabov, A.B., 2019. Length-volume relationship of lake phytoplankton: Length-volume relationship of lake phytoplankton. Limnol. Oceanogr. Methods 17, 58-68.

Murphy, G.E.P., Romanuk, T.N., Worm, B., 2020. Cascading effects of climate change on plankton community structure. Ecol. Evol. 10, 2170-2181.

Orenstein, E.C., Ratelle, D., Briseño-Avena, C., Carter, M.L., Franks, P.J.S., Jaffe, J.S., Roberts, P.L.D., 2020. The Scripps plankton camera system: a framework and platform for in situ microscopy. Limnol. Oceanogr. Methods. https://doi.org/ 10.1002/lom 3.10394 n/a.

Pomati, F., Jokela, J., Simona, M., Veronesi, M., Ibelings, B.W., 2011. An automated platform for phytoplankton ecology and aquatic ecosystem monitoring. Environ. Sci. Technol. 45, 9658-9665.

Pomati, F., Kraft, N.J.B., Posch, T., Eugster, B., Jokela, J., Ibelings, B.W., 2013. Individual cell based traits obtained by scanning flow-cytometry show selection by biotic and abiotic environmental factors during a phytoplankton spring bloom. PLoS One 8, e71677.

Sosik, H.M., Olson, R.J., Armbrust, E.V., 2010. Flow cytometry in phytoplankton research. In: Suggett, D.J., Prášil, O., Borowitzka, M.A. (Eds.), Chlorophyll a Fluorescence in Aquatic Sciences: Methods and Applications. Springer Netherlands, Dordrecht, pp. 171-185.

Sugihara, G., May, R., Ye, H., Hsieh, C.-H., Deyle, E., Fogarty, M., Munch, S., 2012. Detecting causality in complex ecosystems. Science 338, 496-500.

Thomas, M.K., Fontana, S., Reyes, M., Kehoe, M., Pomati, F., 2018. The predictability of a lake phytoplankton community, over time-scales of hours to years. Ecol. Lett. 21, 619-628.

Tollrian, R., Drew Harvell, C., 1999. The Ecology and Evolution of Inducible Defenses. Princeton University Press.

White, E.P., Ernest, S.K.M., Kerkhoff, A.J., Enquist, B.J., 2007. Relationships between body size and abundance in ecology. Trends Ecol. Evol. 22, 323-330.

Xu, F., 2001. Lake ecosystem health assessment: indicators and methods. Water Res. https://doi.org/10.1016/s0043-1354(01)00040-9. 